\title{
The frequency, clinical significance, and pathological features of chronic chorioamnionitis: a lesion associated with spontaneous preterm birth
}

\author{
Chong Jai Kim ${ }^{1,2,3}$, Roberto Romero ${ }^{1,2,4,5}$, Juan Pedro Kusanovic ${ }^{1,2,4}$, Wonsuk Yoo ${ }^{6}$, \\ Zhong Dong ${ }^{1,2}$, Vanessa Topping ${ }^{1,2}$, Francesca Gotsch ${ }^{1,2}$, Bo Hyun Yoon ${ }^{7}$, Je Geun Chi ${ }^{8}$ \\ and Jung-Sun $\mathrm{Kim}^{1,2,3}$
}

${ }^{1}$ Perinatology Research Branch, NICHD/NIH/DHHS, Bethesda, MD, USA; ${ }^{2}$ Perinatology Research Branch, NICHD/ NIH/DHHS, Detroit, MI, USA; ${ }^{3}$ Department of Pathology, Wayne State University School of Medicine, Detroit, MI, USA; ${ }^{4}$ Department of Obstetrics and Gynecology, Wayne State University School of Medicine, Detroit, MI, USA; ${ }^{5}$ Center for Molecular Medicine and Genetics, Wayne State University, Detroit, MI, USA; ${ }^{6}$ Translational Research and Clinical Epidemiology, Department of Internal Medicine, Wayne State University School of Medicine, Detroit, MI, USA; ${ }^{7}$ Department of Obstetrics and Gynecology, Seoul National University College of Medicine, Seoul, Korea and ${ }^{8}$ Department of Pathology, Seoul National University College of Medicine, Seoul, Korea

Acute chorioamnionitis is a well-established lesion of the placenta in cases with intra-amniotic infection. In contrast, the clinicopathological significance of chronic chorioamnionitis is unclear. This study was conducted to determine the frequency and severity of chronic chorioamnionitis in normal pregnancy and in various pregnancy complications. Placentas from the following patient groups were studied: (1) term not in labor $(n=100),(2)$ term in labor $(n=100)$, (3) preterm labor $(n=100)$, (4) preterm prelabor rupture of membranes $(n=100)$, (5) preeclampsia at term $(n=100)$, (6) preterm preeclampsia $(n=100)$, and (7) small-for-gestational-age at term $(n=100)$. Amniotic fluid CXCL10 concentration was measured in 64 patients. CXCL9, CXCL10, and CXCL11 mRNA expressions in the chorioamniotic membranes were assessed using real-time quantitative reverse transcription-PCR. The frequency of chronic chorioamnionitis in the preterm labor group and the preterm prelabor rupture of membranes group was 34 and $39 \%$, respectively, which was higher than that of normal-term placentas (term not in labor, 19\%; term in labor, 8\%; $P<0.05$ each). The frequency of chronic chorioamnionitis in the preeclampsia at term group, preterm preeclampsia group, and small-for-gestational-age group was 23,16 , and $13 \%$, respectively. Concomitant villitis of unknown etiology was found in $\mathbf{3 8}$ and $36 \%$ of preterm labor cases and preterm prelabor rupture of membranes cases with chronic chorioamnionitis, respectively. Interestingly, the median gestational age of preterm chronic chorioamnionitis cases was higher than that of acute chorioamnionitis cases $(P<0.05)$. The median amniotic fluid CXCL10 concentration was higher in cases with chronic chorioamnionitis than in those without, in both the preterm labor group and preterm prelabor rupture of membranes group $(P<0.05$ and $P<0.01$, respectively). CXCL9, CXCL10, and CXCL11 mRNA expression in the chorioamniotic membranes was also higher in cases with chronic chorioamnionitis than in those without chronic chorioamnionitis $(P<0.05)$. We propose that chronic chorioamnionitis defines a common placental pathological lesion among the preterm labor and preterm prelabor rupture of membranes groups, especially in cases of late preterm birth. Its association with villitis of unknown etiology and the chemokine profile in amniotic fluid suggests an immunological origin, akin to transplantation rejection and graftversus-host disease in the chorioamniotic membranes.

Modern Pathology (2010) 23, 1000-1011; doi:10.1038/modpathol.2010.73; published online 26 March 2010

Keywords: chorioamnionitis; amniotic fluid; pregnancy; CXCL9; CXCL10; CXCL11

Correspondence: Dr J-S Kim, MD, PhD, Department of Pathology, Wayne State University School of Medicine, Hutzel Women's Hospital, 3990 John R St, 4 Brush, Detroit, MI 48201, USA. E-mail: jkim@med.wayne.edu or Dr R Romero, MD, Perinatology Research Branch, NICHD, NIH, DHHS, Wayne State University, Hutzel Women's Hospital, 3990 John R St, 4 Brush, Detroit, MI 48201, USA. E-mail: prbchiefstaff@med.wayne.edu

Received 23 December 2009; revised 18 February 2010; accepted 19 February 2010; published online 26 March 2010 
Histopathological examination of human placentas delivered by women with complications of pregnancy often provides valuable and critical information about the mechanisms of disease of the 'Great Obstetrical Syndromes'. ${ }^{1,2}$ Preterm birth affects approximately 500000 newborns every year in the United States, ${ }^{3}$ is the leading cause of perinatal morbidity and mortality worldwide, and is a major cause of chronic illness (eg, cerebral palsy and chronic lung disease).,5 The cost to society is estimated to be 26 billion dollars per year in the United States alone. ${ }^{6,7}$ Preterm birth can result from spontaneous preterm labor/preterm prelabor rupture of membranes or indicated delivery because of maternal (eg, preeclampsia) or fetal (eg, intrauterine growth restriction) indications. ${ }^{1}$

Acute chorioamnionitis is the most common lesion reported in the placenta after spontaneous preterm birth. It is a frequent lesion, both in cases with preterm prelabor rupture of membranes or preterm labor with intact membranes. This lesion is due to microbial invasion of the amniotic cavity documented with cultivation or culture-independent methods. ${ }^{8,9}$ The histopathological features include amniotropic infiltration by both maternal and fetal neutrophils (which are not normally found) in the chorioamniotic membranes and the umbilical cord. ${ }^{10,11}$ This amniotropic neutrophil migration is due to gradients of potent neutrophil chemokines, such as IL-8 and CXCL6 (GCP-2), whose concentrations markedly increase in response to microbial invasion of the amniotic cavity. ${ }^{12,13}$ The clinical significance of the maternal and fetal inflammatory responses in acute chorioamnionitis has been well characterized, and the severity of the lesions is associated with adverse pregnancy outcomes. ${ }^{14,15} \mathrm{~A}$ large body of in vivo and in vitro evidence indicates that the complex of acute chorioamnionitis and intra-amniotic infection is causally linked to preterm labor and preterm prelabor rupture of membranes. ${ }^{16-19}$ Normal spontaneous labor at term in the absence of histological chorioamnionitis also has a molecular signature of acute inflammation, suggesting that inflammation is a phenomenon of both physiological and pathological human parturition. ${ }^{20}$

Chronic chorioamnionitis is defined by the infiltration of lymphocytes in the chorioamniotic membranes and the chorionic plate, similar to that of neutrophils in acute chorioamnionitis. ${ }^{21}$ The original description and characterization of this lesion represents a series of seminal contributions. ${ }^{21-23}$ Chronic chorioamnionitis has been associated with previous spontaneous abortion, intrauterine growth restriction, and preterm birth. It is of interest that chronic chorioamnionitis is often associated with 'chronic villitis of unknown etiology.' Gersell et al ${ }^{22}$ reported villitis of unknown etiology in $65 \%$ of cases (11/17 chronic chorioamnionitis cases), whereas Jacques and Qureshi ${ }^{21}$ reported the coexistence of these lesions in $71 \%$ of cases $(22 / 31$ chronic chorioamnionitis cases). However, the clinical significance, underlying pathophysiology, and frequency of chronic chorioamnionitis have not been clearly defined because the number of cases reported has been limited. The purpose of this study was to determine the frequency and clinical significance of this unique lesion. We conducted a systematic study of 700 cases of patients with normal pregnancies as well as those with preterm labor, preterm prelabor rupture of membranes, preeclampsia, and small-forgestational-age neonates. As there is an association between chronic chorioamnionitis and villitis of unknown etiology, we postulated that dysregulated expression of anti-angiogenic T-cell chemokines CXCL9, CXCL10, and CXCL11 in the amniotic fluid and the chorioamniotic membranes may, in part, be responsible for this lesion. ${ }^{24}$ Therefore, we performed a histopathological review of the placentas and the chorioamniotic membranes and determined the expression of T-cell chemokines in the amniotic fluid (CXCL10) and in the chorioamniotic membranes (CXCL9, CXCL10, and CXCL11).

\section{Materials and methods}

\section{Study Population}

Placental tissues were collected at the time of delivery. All participating patients provided written informed consent, and the Institutional Review Board of the Eunice Kennedy Shriver National Institute of Child Health and Human Development, National Institutes of Health, US Department of Health and Human Services, approved the collection and use of biological materials for research purposes. Using the database of the Bank of Biological Materials of the Perinatology Research Branch of the Eunice Kennedy Shriver National Institute of Child Health and Human Development, we identified 700 cases presenting with the following diagnoses: (1) women not in labor at term without pregnancy complications $(n=100)$, (2) women in labor at term $(n=100)$, (3) patients in preterm labor with intact membranes $(n=100),(4)$ patients with preterm prelabor rupture of membranes $(n=100)$, (5) patients with preeclampsia at term $(n=100),(6)$ patients with preterm preeclampsia $(n=100)$, and (7) patients with small-for-gestational-age neonates born at term $(n=100)$. Preterm labor was defined by the presence of regular uterine contractions with cervical dilation that led to delivery before 37 weeks of gestation. Preeclampsia was defined by both hypertension (systolic blood pressure $\geq 140 \mathrm{~mm} \mathrm{Hg}$ or diastolic blood pressure $\geq 90 \mathrm{~mm} \mathrm{Hg}$ on at least two occasions, $4 \mathrm{~h}$ to 1 week apart) and proteinuria $(\geq 300 \mathrm{mg}$ in a 24 -h urine collection or one dipstick measurement $\geq 2+$ ). Small-for-gestational-age was defined as $<10^{\text {th }}$ percentile in birth weight for gestational age. Amniotic fluid samples $(n=64)$ were obtained by transabdominal amniocentesis from a select group of 
women who underwent amniocentesis for clinical indications. Samples were kept at $-80^{\circ} \mathrm{C}$ until use in the CXCL10 assay. Extraplacental chorioamniotic membranes $(n=59)$ had been collected in RNAlater (Qiagen, Valencia, CA, USA), and kept at $-80{ }^{\circ} \mathrm{C}$ until use.

\section{Histopathological Examination}

In each case, hematoxylin and eosin-stained sections of the chorioamniotic membranes roll $(n=1)$, umbilical cord $(n=1)$, and placental disc $(n=3)$ were examined. Pathologists were masked to the clinical diagnosis except for the gestational age at delivery. The diagnosis of chronic chorioamnionitis was made when lymphocytic infiltration into the chorionic trophoblast layer or chorioamniotic connective tissue was observed. The severity of chronic chorioamnionitis was scored based upon two parameters. The extent of inflammation was graded 0 when there was no inflammation, 1 when there were more than two foci of or patchy inflammation, and 2 when diffuse inflammation was present. The stage of inflammation was scored as stage 1 if amniotropic lymphocytic infiltration was limited to the chorionic trophoblast layer sparing the chorioamniotic connective tissue, and stage 2 if lymphocytic infiltration into the chorioamniotic connective tissue was noted. Histopathological screening for other lesions of the placenta was performed according to the diagnostic criteria proposed by the Perinatal Section of the Society for Pediatric Pathology. Such classification encompasses lesions consistent with amniotic fluid infection, maternal vascular underperfusion, and fetal vascular obstruction. ${ }^{10,25,26}$ The diagnosis of chronic deciduitis with plasma cells was given when lymphoplasmacytic infiltrate was present in the decidua of the basal plate. ${ }^{27}$

\section{Immunohistochemistry}

Immunohistochemistry was performed to identify T-cell infiltration by using an antibody against CD3 in all patients with preterm prelabor rupture of membranes $(n=100)$. Formalin-fixed, paraffin-embedded, 5- $\mu \mathrm{m}$-thick tissue sections of the chorioamniotic membranes were placed on silanized slides and stained using a Ventana Discovery automatic staining system (Ventana Medical Systems, Tucson, AZ, USA). Immunostaining was performed using mouse monoclonal anti-CD3 antibody (1:50, Novocastra, Newcastle, UK). The Discovery DAB Map Kit (Ventana Medical Systems) was used to detect the chromogen reaction of horseradish peroxidase.

\section{Enzyme-Linked Immunosorbent Assay}

Amniotic fluid samples obtained by transabdominal amniocentesis were centrifuged at $1300 \times g$ for
10 min and stored at $-80^{\circ} \mathrm{C}$ until use. The amniotic fluid concentration of CXCL10 was measured by specific enzyme-linked immunosorbent assay (R\&D Systems, Minneapolis, MN, USA) according to the manufacturer's instructions. The sensitivity of the assay was $4.22 \mathrm{pg} / \mathrm{ml}$. The inter-assay coefficient of variation was $5.3 \%$, and the intra-assay coefficient of variation was $3.56 \%$.

\section{Real-Time Quantitative Reverse Transcription- Polymerase Chain Reaction (qRT-PCR)}

Chorioamniotic membranes collected in RNAlater from patients with preterm labor and preterm prelabor rupture of membranes were available in 59 cases. Total RNA was isolated using Trizol, and further purified and DNased using an RNeasy minicolumn (Qiagen). Reverse transcription reaction was performed using SuperScript III reverse transcriptase (Invitrogen, Carlsbad, CA, USA) and oligodT primers with $100 \mathrm{ng}$ of total RNA. PCR analyses were performed with TaqMan Gene Expression Assays (CXCL9, Hs00171065_m1; CXCL10, Hs00171042_m1; CXCL11, Hs00171138_m1; Applied Biosystems, Foster City, CA, USA). RPLPO was used for normalization. The ABI 7500 FAST Real Time PCR system was used for PCR reactions.

\section{Statistical Analysis}

Means and s.d. were calculated for continuous variables whereas frequencies and percentages were reported for categorical variables. Unpaired $t$-tests and $\chi^{2}$ tests were applied for continuous and discrete variables, respectively. Analysis of variance (ANOVA) was performed to compare differences in the mean expression of CXCL9 mRNA, CXCL10 mRNA, and CXCL11 mRNA in the chorioamniotic membranes according to grade, stage, and severity of chronic chorioamnionitis. If the overall test was significant, multiple comparisons (post hoc tests) were performed to determine the source of the difference. Distributions of continuous variables were examined for skewness and normality using Kolmogorov-Smirnov tests. If the data were far from the normality, a generalized linear model using the ranked data was applied. Statistical analyses were performed using SAS Version 9.2 (Statistical Analysis Software, Cary, NC, USA), and all $P$-values were two-sided. $P$-values of $<0.05$ were considered to indicate statistical significance.

\section{Results}

Frequency and Severity of Chronic Chorioamnionitis

The demographic characteristics and the frequency of chronic chorioamnionitis in each group are shown in Table 1. Examples of lymphocytic infiltration limited to the chorionic trophoblast layer 
Table 1 Patient demographics, clinical characteristics, and frequency of chronic chorioamnionitis in each group

\begin{tabular}{lcccc}
\hline Group & $\begin{array}{c}\text { Maternal age } \\
\text { (years, mean } \pm \text { s.d.) }\end{array}$ & $\begin{array}{c}\text { Gestational age at delivery } \\
\text { (weeks, mean } \pm \text { s.d.) }\end{array}$ & $\begin{array}{c}\text { Birth weight } \\
\text { (g, mean } \pm \text { s.d.) }\end{array}$ & $\begin{array}{c}\text { Labor (absent/ } \\
\text { spontaneous/induced) }\end{array}$ \\
\hline TNL & $29.4 \pm 5.9$ & $39.5 \pm 1.3$ & $3449.8 \pm 314.4$ & $100 / 0 / 0$ \\
of CCA (\%)
\end{tabular}

CCA: chronic chorioamnionitis; TNL: term not in labor; TIL: term in labor; PTL: preterm labor; PPROM: preterm prelabor rupture of membranes; TPE: preeclampsia at term; PPE: preterm preeclampsia; SGA: small-for-gestational-age at term.
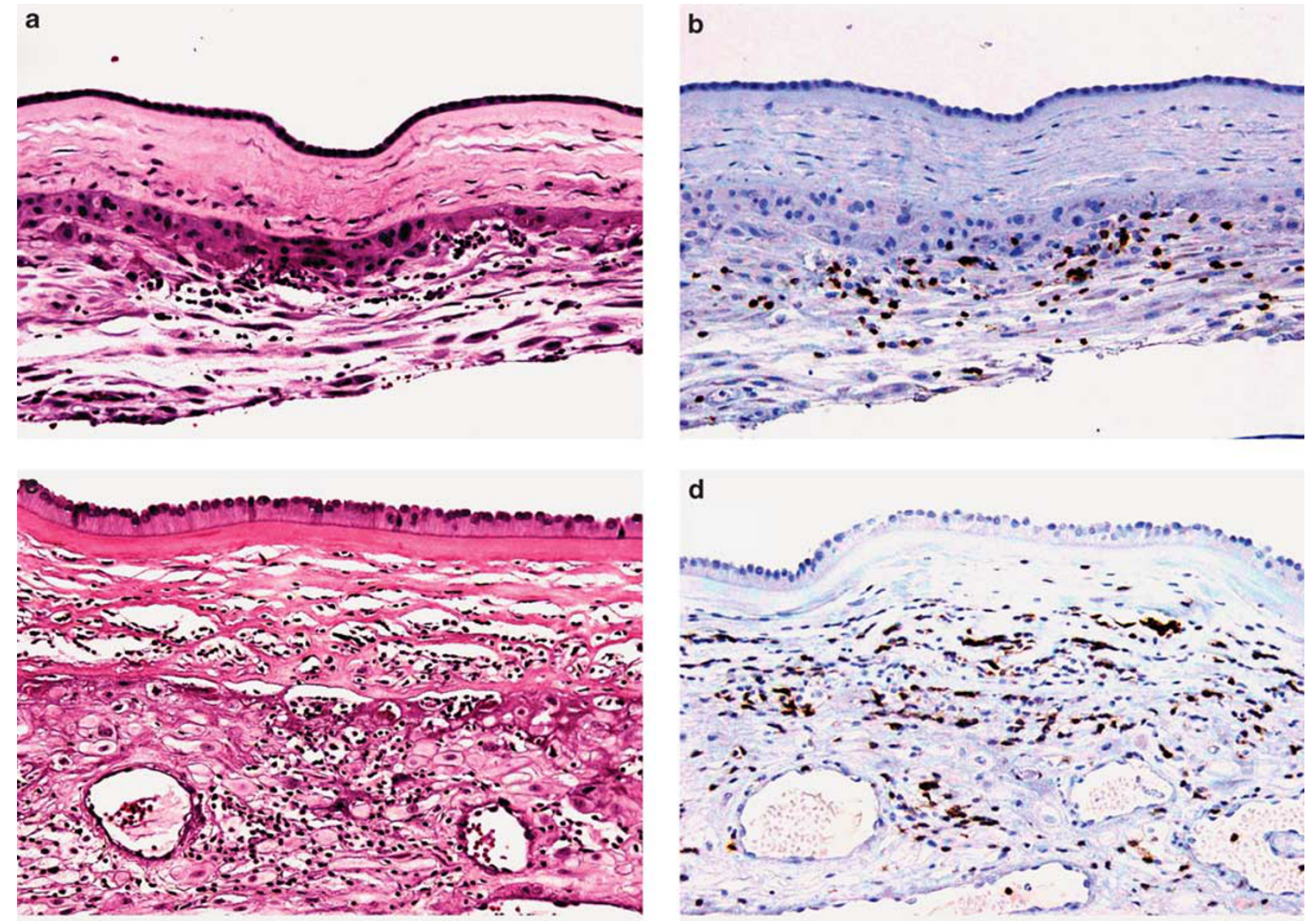

Figure 1 Histological characteristics of chronic chorioamnionitis. (a, b) Stage 1 inflammation showing infiltration of lymphocytes limited to the chorionic trophoblast layer (a). CD3 immunostaining shows that the majority of these cells are T cells (b). (c, d) Stage 2 inflammation is characterized by infiltration of lymphocytes into the chorioamniotic connective tissue layer (H\&E, c), which are largely CD3 + T cells $(\mathbf{d})$.

(stage 1) and into the chorioamniotic connective tissue (stage 2) are shown in Figure 1. Chronic chorioamnionitis was commonly associated with destruction and thinning of the chorionic trophoblast layer. The frequency of chronic chorioamnionitis was: $19 \%(19 / 100)$ for women at term not in labor, $8 \%(8 / 100)$ for women at term in labor, $34 \%$ $(34 / 100)$ for women in preterm labor with intact membranes, $39 \%$ (39/100) for women with preterm prelabor rupture of membranes, $23 \%$ (23/100) for women with preeclampsia at term, 16\% (16/100) for women with preterm preeclampsia, and 13\% (13/ 100) for women with small-for-gestational-age neonates born at term (Figure 2). Chronic chorioamnionitis was more common in preterm labor and preterm prelabor rupture of membranes cases than in term not in labor $(P<0.05$ and $P<0.01$, respectively) or term in labor $(P<0.001$ for each) cases. The 


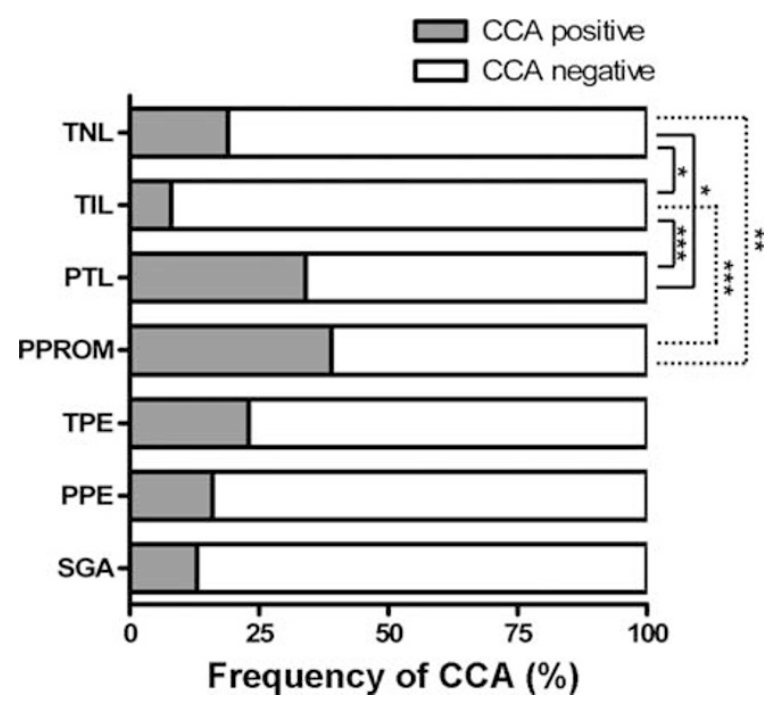

Figure 2 Frequency of chronic chorioamnionitis (CCA) in different obstetrical settings. Frequency of chronic chorioamnionitis in the preterm labor (34\%) and preterm prelabor rupture of membranes (39\%) groups are significantly higher when compared with the term not in labor $(19 \%)$ and term in labor (8\%) groups. The difference in frequency between term not in labor and term in labor cases was also statistically significant. The frequency of chronic chorioamnionitis in each group ranged from 8 to $39 \%$. TNL: term not in labor; TIL: term in labor; PTL: preterm labor; PPROM: preterm prelabor rupture of membranes; TPE: preeclampsia at term; PPE: preterm preeclampsia; SGA: small-forgestational-age at term. ${ }^{*} P<0.05,{ }^{* *} P<0.01,{ }^{*}{ }^{*} P<0.001$.

frequency of chronic chorioamnionitis in term in labor cases was significantly lower than that in term not in labor and preeclampsia at term cases $(P<0.05$ and $P<0.01$, respectively). The frequency of chronic chorioamnionitis in preterm labor and preterm prelabor rupture of membranes cases was significantly higher than that observed in preterm preeclampsia cases $(P<0.01$ and $P<0.001$, respectively). Interestingly, placentas from patients with preterm prelabor rupture of membranes tended to have higher stage chronic chorioamnionitis when compared with preterm labor cases $(P<0.06)$, although there was no difference in the frequency of chronic chorioamnionitis between the two groups.

Other pathological changes observed in the placentas of each group are summarized in Table 2. The frequency of villitis of unknown etiology among the groups ranged between 13\% (preterm preeclampsia group) and $25 \%$ (preeclampsia at term group; Figure 3a). Placentas with chronic chorioamnionitis in patients with preterm labor and preterm prelabor rupture of membranes had concomitant villitis of unknown etiology in 38 and $36 \%$ of cases, respectively, whereas villitis of unknown etiology was found in only 9 and $7 \%$ of the placentas in the same diagnostic group without chronic chorioamnionitis (Figure 3b). The frequency of villitis of unknown etiology in cases with chronic chorioamnionitis varied among the groups: $37 \%$ in term not in labor, $38 \%$ in term in labor, $44 \%$ in preeclampsia at term, $19 \%$ in preterm preeclampsia, and $31 \%$ in small-forgestational-age neonates. Thus, among patients with chronic chorioamnionitis, the frequency of villitis of unknown etiology was high.

There was also a significant difference in the frequency of chronic deciduitis with plasma cells among the groups $(P<0.05)$. The frequency of chronic deciduitis with plasma cells was $13 \%$ for women at term not in labor, $11 \%$ for women at term in labor, $22 \%$ for women in preterm labor with intact membranes, $25 \%$ for women with preterm prelabor rupture of membranes, $9 \%$ for women with preeclampsia at term, $16 \%$ for women with preterm preeclampsia, and $14 \%$ for women with small-forgestational-age neonates born at term. Its frequency was higher in cases with spontaneous preterm birth (preterm labor and preterm prelabor rupture of membranes) than in those who delivered at term (term not in labor and term in labor) (24 vs $12 \%$, $P<0.005)$. The frequency was significantly higher in cases with chronic chorioamnionitis or villitis of unknown etiology than in cases without (for chronic chorioamnionitis: 39 vs $9 \%$; for villitis of unknown etiology: 38 vs 11\%, $P<0.001$ for each). Chronic deciduitis with plasma cells was largely associated with basal villitis in each placenta as has been described previously. ${ }^{28}$

Acute chorioamnionitis was found in 22 and 19\% of preterm labor and preterm prelabor rupture of membranes cases, respectively (see Table 2). Interestingly, the mean gestational age at delivery of cases with chronic chorioamnionitis was significantly higher than that of acute chorioamnionitis (33.9 weeks vs 32.4 weeks; $P<0.05$ ) in preterm labor and preterm prelabor rupture of membranes cases. In nine cases, lesions of acute chorioamnionitis and chronic chorioamnionitis were observed in the same placenta, showing 'acute on chronic' chorioamnionitis as previously described. ${ }^{22}$

Histopathological features consistent with maternal vascular underperfusion or fetal vascular obstruction were also variably found among the groups analyzed (Table 2). The frequency of lesions considered to represent maternal vascular underperfusion were more common in preterm preeclampsia cases than in preterm labor and preterm prelabor rupture of membranes cases $(P<0.001$ for each). There was no difference in the frequency of maternal vascular underperfusion or fetal vascular obstruction between cases with and without chronic chorioamnionitis.

To assess the correlation between the histological evaluation of chronic chorioamnionitis based on hematoxylin and eosin staining and the immunostaining for $\mathrm{T}$ cells, we performed immunostaining for CD3 in all placentas of patients with preterm prelabor rupture of membranes, because chronic chorioamnionitis was found most frequently in this group. A masked review of CD3 immunoreactivity 
Table 2 Histological findings in each group with and without chronic chorioamnionitis

\begin{tabular}{|c|c|c|c|c|c|}
\hline Group & $\begin{array}{l}\text { Number } \\
\text { of cases }\end{array}$ & $\begin{array}{l}\text { Villitis of unknown } \\
\text { etiology }\end{array}$ & $\begin{array}{c}\text { Acute } \\
\text { chorioamnionitis }\end{array}$ & $\begin{array}{l}\text { Maternal vascular } \\
\text { underperfusion }\end{array}$ & $\begin{array}{c}\text { Fetal vascular } \\
\text { thrombo-occlusive disease }\end{array}$ \\
\hline \multicolumn{6}{|l|}{$T N L$} \\
\hline CCA negative & 81 & $10(12.3 \%)$ & $4(4.9 \%)$ & $6(7.4 \%)$ & $0(0.0 \%)$ \\
\hline CCA positive & 19 & $7(36.8 \%)$ & $1(5.3 \%)$ & $1(5.3 \%)$ & $0(0.0 \%)$ \\
\hline Total & 100 & $17(17 \%)$ & $5(5 \%)$ & $7(7 \%)$ & $0(0.0 \%)$ \\
\hline \multicolumn{6}{|l|}{ TIL } \\
\hline CCA negative & 92 & $11(12.0 \%)$ & $21(22.8 \%)$ & $3(3.3 \%)$ & $0(0.0 \%)$ \\
\hline CCA positive & 8 & $3(37.5 \%)$ & $1(12.5)$ & $1(12.5 \%)$ & $0(0.0 \%)$ \\
\hline Total & 100 & $14(14 \%)$ & $22(22 \%)$ & $4(4 \%)$ & $0(0.0 \%)$ \\
\hline \multicolumn{6}{|l|}{ PTL } \\
\hline CCA negative & 66 & $6(9.1 \%)$ & $20(30.3 \%)$ & $11(16.7 \%)$ & $1(1.5 \%)$ \\
\hline CCA positive & 34 & $13(38.2 \%)$ & $2(5.9 \%)$ & $5(14.7 \%)$ & $1(2.9 \%)$ \\
\hline Total & 100 & $19(19 \%)$ & $22(22 \%)$ & $16(16 \%)$ & $2(2 \%)$ \\
\hline \multicolumn{6}{|l|}{ PPROM } \\
\hline CCA negative & 61 & $4(6.6 \%)$ & $12(19.7 \%)$ & $5(8.2 \%)$ & $0(0.0 \%)$ \\
\hline CCA positive & 39 & $14(35.9 \%)$ & $7(17.9 \%)$ & $5(12.8 \%)$ & $1(2.6 \%)$ \\
\hline Total & 100 & $18(18 \%)$ & $19(19 \%)$ & $10(10 \%)$ & $1(1.0 \%)$ \\
\hline \multicolumn{6}{|l|}{ TPE } \\
\hline CCA negative & 77 & $15(19.5 \%)$ & $7(9.1 \%)$ & $11(14.3 \%)$ & $1(1.3 \%)$ \\
\hline CCA positive & 23 & $10(43.5 \%)$ & $3(13.0 \%)$ & $8(34.8 \%)$ & $0(0.0 \%)$ \\
\hline Total & 100 & $25(25 \%)$ & $10(10 \%)$ & $19(19 \%)$ & $1(1.0 \%)$ \\
\hline \multicolumn{6}{|l|}{$P P E$} \\
\hline CCA negative & 84 & $10(11.9 \%)$ & $1(1.2 \%)$ & $53(63.1 \%)$ & $1(1.2 \%)$ \\
\hline CCA positive & 16 & $3(18.8 \%)$ & $0(0 \%)$ & $8(50.0 \%)$ & $0(0.0 \%)$ \\
\hline Total & 100 & $13(13 \%)$ & $1(1 \%)$ & $61(61 \%)$ & $1(1 \%)$ \\
\hline \multicolumn{6}{|l|}{$S G A$} \\
\hline CCA negative & 87 & $18(20.7 \%)$ & $7(8.0 \%)$ & $14(16.1 \%)$ & $1(1.1 \%)$ \\
\hline CCA positive & 13 & $4(30.8 \%)$ & $0(0 \%)$ & $2(15.4 \%)$ & $1(7.7 \%)$ \\
\hline Total & 100 & $22(22 \%)$ & $7(7 \%)$ & $16(16 \%)$ & $2(2 \%)$ \\
\hline
\end{tabular}

CCA: chronic chorioamnionitis; TNL: term not in labor; TIL: term in labor; PTL: preterm labor; PPROM: preterm prelabor rupture of membranes; TPE: preeclampsia at term; PPE: preterm preeclampsia; SGA: small-for-gestational-age at term.

revealed chronic chorioamnionitis in $54 \%$ of preterm prelabor rupture of membranes cases. CD3 + T-lymphocytic infiltration that met criteria used in this study was additionally detected in 15 of 61 non-chronic chorioamnionitis cases classified by histology alone. This was because of differences in microscopic planes from original hematoxylin and eosin slides, addition of mild (grade 1/stage 1) cases, grade 1/stage 2 lesions in which $\mathrm{T}$ cells were unexpectedly found in the chorioamniotic connective tissue by CD3 immunostaining, and a grade 2/stage 2 lesion in which lymphocytic infiltration was obscure in hematoxylin and eosin staining because of the presence of concomitant acute chorioamnionitis.

\section{Amniotic Fluid CXCL10 Concentration and CXCL9, CXCL10, and CXCL11 mRNA Expressions in the Chorioamniotic Membranes}

As chronic chorioamnionitis shows an amniotropic T-cell migration pattern, we postulated that the concentrations of amniotic fluid T-cell chemokines CXCL9, CXCL10, and CXCL11 would be higher in chronic chorioamnionitis cases, as placental expression of those chemokines was increased in villitis of unknown etiology cases. ${ }^{24}$ Amniotic fluid samples obtained by amniocentesis within 4 weeks before delivery were available in 64 preterm labor and preterm prelabor rupture of membranes cases. Comparisons between cases with and without chronic chorioamnionitis showed that cases with chronic chorioamnionitis had significantly higher amniotic fluid CXCL10 concentration $(P<0.01$; Figure 4a). There was a relationship between the severity of chronic chorioamnionitis and the amniotic fluid CXCL10 concentration among grade 1/stage 1, grade 2 /stage 1 or grade 1/stage 2 , and grade 2 /stage 2 cases. This clearly showed that the severity of chronic chorioamnionitis relates to the concentration of amniotic fluid CXCL10 $(P<0.001, R=0.446)$. On the other hand, amniotic fluid CXCL10 concentration was not significantly different in acute chorioamnionitis, whereas it seemed to be elevated in villitis of unknown etiology cases, although the number of cases with 
a
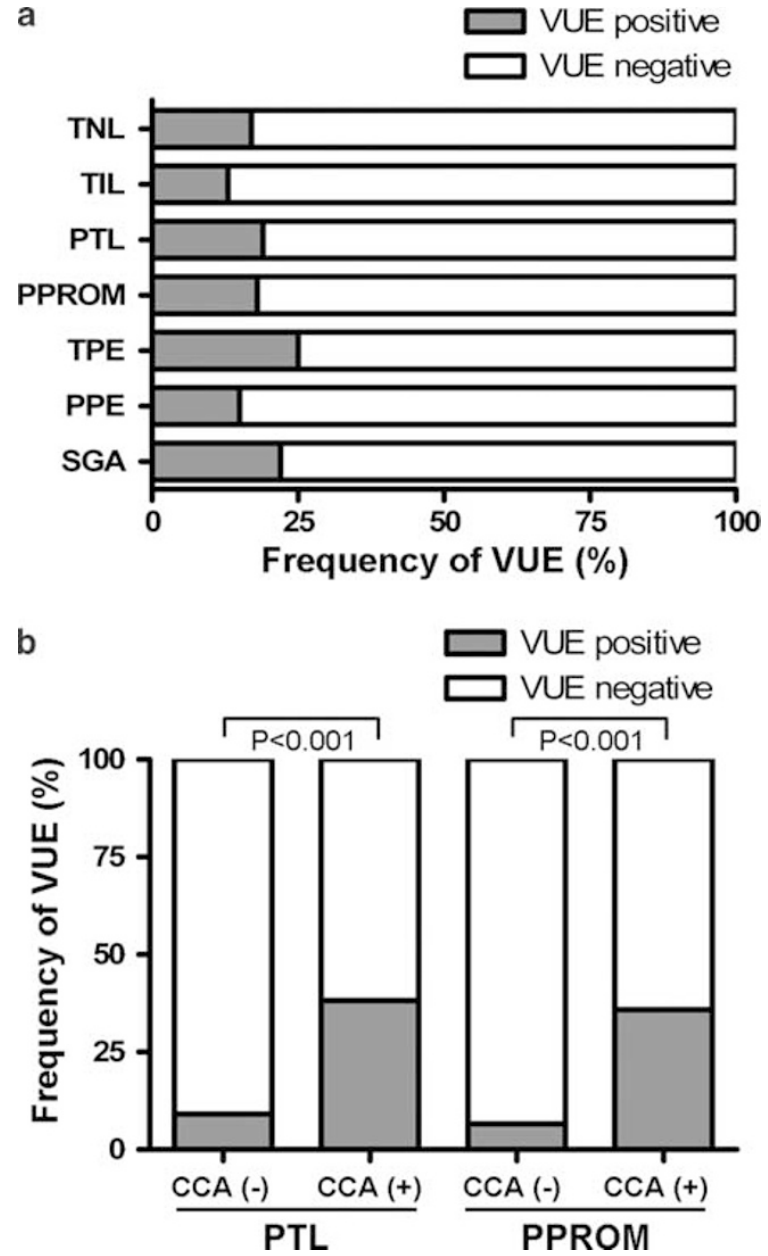

Figure 3 Frequency of villitis of unknown etiology (VUE) in each group with chronic chorioamnionitis (CCA). (a) Frequency of villitis of unknown etiology in each group ranged from $13 \%$ (preterm preeclampsia group) to $25 \%$ (preeclampsia at term group). (b) Concomitant villitis of unknown etiology was found in 38 and $36 \%$ of preterm labor and preterm prelabor rupture of membranes cases with chronic chorioamnionitis, respectively, but in 9 and $7 \%$ of preterm labor and preterm prelabor rupture of membranes cases without chronic chorioamnionitis, respectively. TNL: term not in labor; TIL: term in labor; PTL: preterm labor; PPROM: preterm prelabor rupture of membranes; TPE: preeclampsia at term; PPE: preterm preeclampsia; SGA: small-forgestational-age at term.

isolated villitis of unknown etiology without chronic chorioamnionitis $(n=3)$ was insufficient for meaningful statistical comparison (Figure 4b).

In a previous analysis of the human amnion transcriptome, we found mRNA expression of CXCL9, CXCL10, and CXCL11. ${ }^{29}$ As the chorioamniotic membranes are potential sources of amniotic fluid CXCL9, CXCL10, and CXCL11, we tested whether mRNA expression of these chemokines in the chorioamniotic membranes changed in chronic chorioamnionitis. qRT-PCR analysis showed that mRNA expression of these three chemokines was significantly higher in the chorioamniotic membranes with chronic chorioamnionitis than in those without chronic chorioamnionitis in preterm labor and preterm prelabor rupture of membranes cases. The median mRNA expressions of CXCL9, CXCL10, and CXCL11 in chronic chorioamnionitis cases were 2.64-fold, 1.69-fold, and 1.74-fold higher than in cases without chronic chorioamnionitis $(P<0.01$, $P<0.05$, and $P<0.05$, respectively; Figures 5a-c). However, mRNA expressions of these chemokines did not increase with acute chorioamnionitis.

On further examination of amniotic fluid CXCL10 concentration and mRNA expressions of CXCL9, CXCL10, and CXCL11 in the chorioamniotic membranes according to the severity of chronic chorioamnionitis, we found that even histologically mild chronic chorioamnionitis had significantly higher amniotic fluid CXCL10 concentration $(P<0.05)$ and higher expression of CXCL9 mRNA $(P<0.05)$ in the chorioamniotic membranes than in those without chronic chorioamnionitis (Figures 6a-d).

\section{Discussion}

The primary finding of this study is that chronic chorioamnionitis is a common histological lesion of the placenta among patients with preterm labor with intact membranes and preterm prelabor rupture of membranes. Moreover, the changes of amniotic fluid CXCL10 concentration and the common association with villitis of unknown etiology indicate that chronic chorioamnionitis is not simply a locally restricted histological phenomenon. The chronic chorioamnionitis and elevated amniotic fluid T-cell chemokine CXCL10 complex could be considered a counterpart of the acute chorioamnionitis and elevated neutrophil chemokines (IL-8 and CXCL6) complex, which is due to ascending intra-amniotic infection. ${ }^{12,13}$ The median gestational age of chronic chorioamnionitis in preterm labor and preterm prelabor rupture of membranes groups is higher than that of acute chorioamnionitis. This is important because the pathological basis for spontaneous preterm labor and preterm prelabor rupture of membranes resulting in 'late preterm (near-term) delivery' has remained a mystery. ${ }^{7,30}$ Our data suggest that this is an important lesion that accounts for the most frequent type of all spontaneous preterm deliveries. This association is novel.

In contrast to acute chorioamnionitis after microbial invasion of the amniotic cavity, the underlying etiology of chronic chorioamnionitis is unknown. Subclinical intra-amniotic infection by microorganisms (such as viruses) is clearly one possibility. Several studies focusing on the viral load of amniotic fluid using PCR techniques have reported variable detection rates for viruses, such as cytomegalovirus, parvovirus, and adenovirus. ${ }^{31,32}$ Miller et $a l^{33}$ even detected viral genomes in 40 of 686 cases of second-trimester genetic amniocentesis obtained from asymptomatic patients. However, meticulous evaluation for potential viral, bacterial, and protozoal pathogens did not reveal any specific infectious 

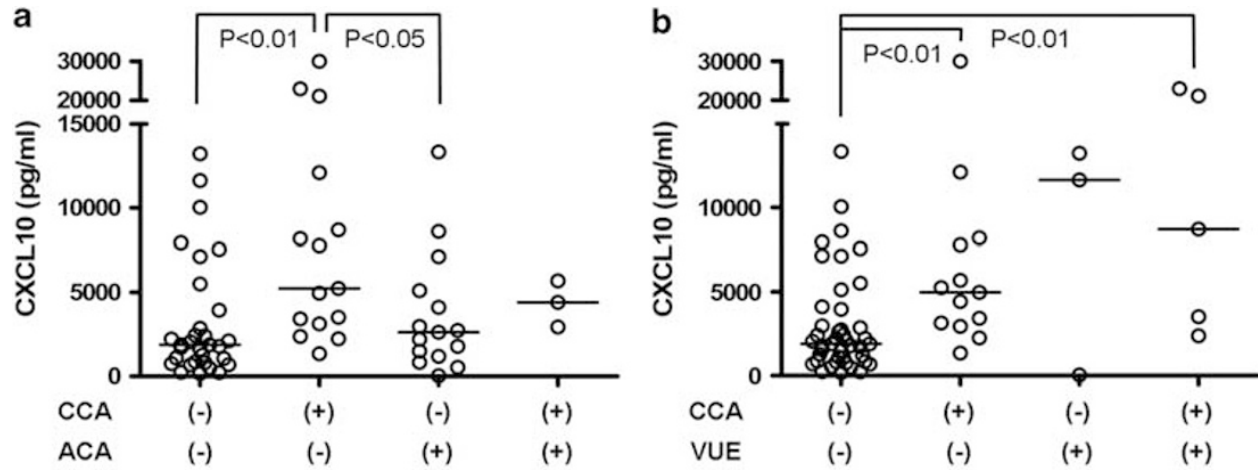

Figure 4 Comparisons of amniotic fluid CXCL10 according to the presence of chronic chorioamnionitis (CCA), acute chorioamnionitis (ACA), and villitis of unknown etiology (VUE). (a) Although chronic chorioamnionitis cases have significantly higher amniotic fluid CXCL10 concentration, it is not elevated in cases with acute chorioamnionitis. (b) Chronic chorioamnionitis cases with concomitant villitis of unknown etiology have higher amniotic fluid CXCL10 concentrations when compared with cases without inflammation.

agents in a previous analysis of 17 cases of chronic chorioamnionitis. ${ }^{22}$ Jacques and Qureshi ${ }^{21}$ also pointed out that the pathology of villitis lesions was not consistent with the typical presentation found in villitis of infectious origins, such as cytomegalovirus, toxoplasmosis, syphilis, and herpes simplex virus. Therefore, the frequent coexistence of chronic chorioamnionitis and villitis of unknown etiology noted in previous studies (and documented in the current study) strongly suggests that these two pathological lesions are likely to have a non-infectious origin. We propose, based on the change in the chemokine profile of amniotic fluid and the association with villitis of unknown etiology, an immunological etiology. Specifically, we postulate that chronic chorioamnionitis is akin to combined allograft rejection and graft-versus-host disease in the chorioamniotic membranes as previously suggested for villitis of unknown etiology. ${ }^{24}$ The essential pathology of villitis of unknown etiology is the interaction between infiltrating maternal $\mathrm{T}$ cells and fetal placental resident macrophages (Hofbauer cells) in the fetal chorionic villi. $^{34,35}$ We think that the pathophysiology of chronic chorioamnionitis is basically identical, given that the cells involved include maternal T cells, fetal chorionic trophoblasts, Hofbauer cells, and myofibroblasts in the chorioamniotic connective tissue.

Multiple etiologies such as infection, uteroplacental ischemia, uterine over-distension, and abnormal allograft rejection are thought to be associated with preterm birth. ${ }^{2,36}$ Therefore, preterm parturition itself has a syndromic nature. ${ }^{2}$ Ascending intrauterine infection and uteroplacental ischemia are typical histopathological alterations of the placenta found in major subsets of preterm birth. ${ }^{10,26,37,38}$ Acute chorioamnionitis is the pathological expression of intra-amniotic infection. Histological features compatible with inadequate, superficial placentation and subsequent underperfusion of the placenta characterize another group of preterm birth, including preeclampsia and intrauterine growth restriction before the gestational age of 28 weeks. ${ }^{39}$ Our observations clearly indicate that chronic chorioamnionitis comprises a major group, a definable placental phenotype associated with preterm birth. Given that this lesion is observed at a higher gestational age at birth than others, we postulate that this lesion is a major feature in late preterm (near-term) birth. We found the trajectory of the frequency of chronic chorioamnionitis-decreasing with advancing gestationparticularly intriguing. Villitis of unknown etiology is considered a lesion of term (not preterm) placentas; ${ }^{28,40}$ therefore, this lesion cannot account for the pathology of late preterm (near-term) birth. The underlying pathological lesion of late preterm birth is of major health-care importance because these are the most common types of all preterm births, and infants have an increased rate of mortality, respiratory distress, infection, and behavioral problems despite being born so close to term. ${ }^{41,42}$

A unique finding of this study is a surprisingly high frequency of chronic chorioamnionitis in each group. It seems that the detection of mild chronic chorioamnionitis lesions (which are likely to be underdiagnosed in routine surgical pathology practice) is primarily responsible for this observation. Gersell et $a l^{22}$ provided meticulous and comprehensive descriptions of the pathology of chronic chorioamnionitis based on the types of leukocytic infiltrates (lymphocytic, lymphoplasmacytic, and neutrophilic) and the anatomical regions (chorion alone, amnion and chorion, and chorionic plate) involved. Jacques and Qureshi ${ }^{21}$, in their analysis of chronic chorioamnionitis cases, applied a grading system to describe the extent of inflammation $(1+$, few scattered foci; $2+$, up to half of the membrane roll involved; and $3+$, more than half of the membrane roll involved). Our criteria for the grading and staging of inflammation reflect the principles used in both of the studies, the primary difference 

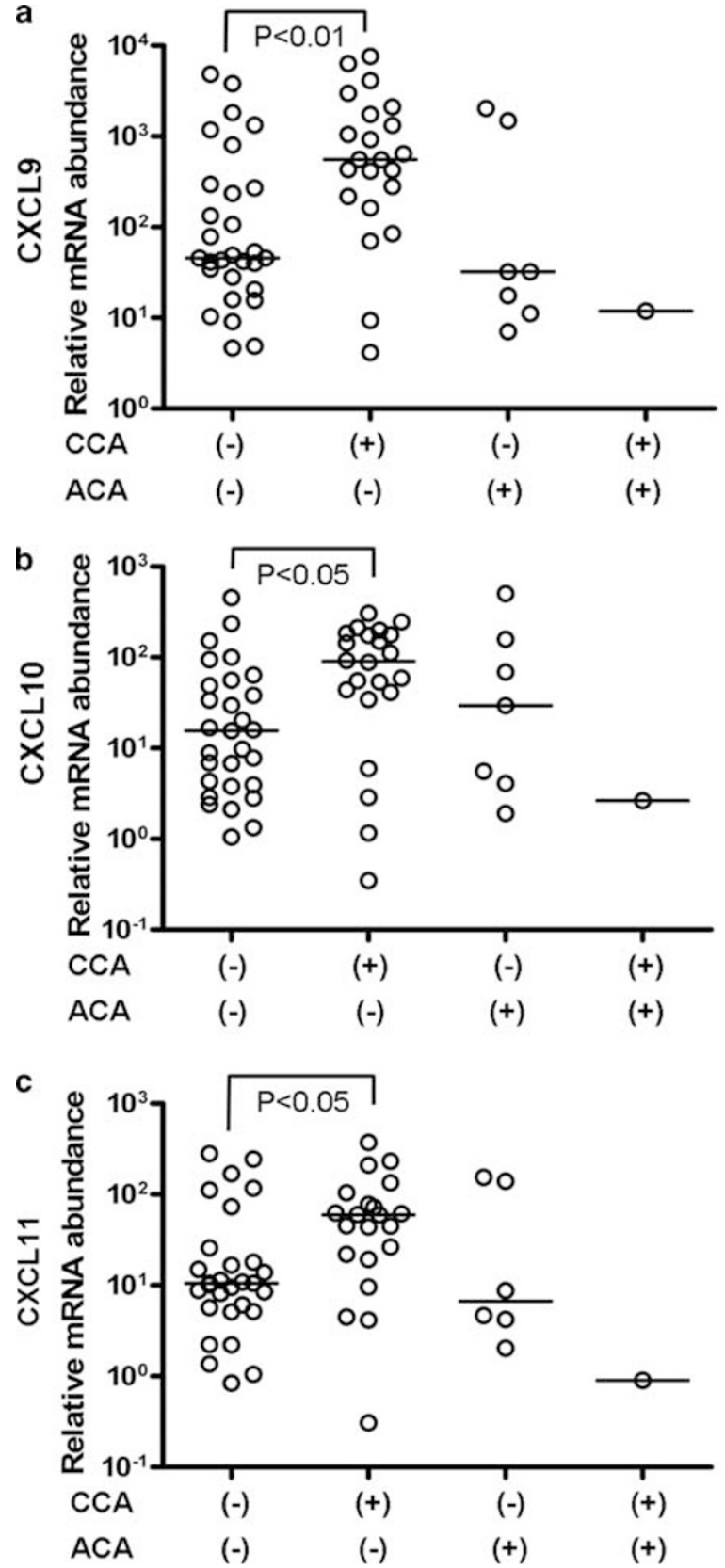

Figure 5 mRNA expressions of CXCL9, CXCL10, and CXCL11 in the chorioamniotic membranes. CXCL9 (a), CXCL10 (b), and CXCL11 (c) mRNA expressions are significantly higher in chronic chorioamnionitis (CCA)-positive cases, but not in acute chorioamnionitis (ACA)-positive cases when compared with cases without inflammation.

being the inclusion of stage 1 lesions in which inflammatory infiltrates are confined to the chorionic trophoblast layer. Therefore, the pathological significance of mild chronic chorioamnionitis could be a subject of debate if it were based on morphologic grounds alone; however, the clear changes in amniotic fluid concentrations of CXCL10 and increased CXCL9, CXCL10, and CXCL11 mRNA expression in the chorioamniotic membranes lends strong support for its biological significance, even in mild cases. We argue that this lesion reflects a change in the profile of intra-amniotic chemokines. This situation is analogous to elevation of amniotic fluid IL-6 in even mild acute chorioamnionitis. ${ }^{43}$

Of note, two recent studies reported the presence of chronic inflammation and chronic deciduitis in substantial proportions of preterm cases. Goldenberg et $a l^{44}$ found chronic inflammation involving any site of the placenta (free fetal membranes, chorionic plate, and decidua basalis) in 21 and $13 \%$ of indicated and spontaneous preterm birth cases, respectively. On the basis of the pathological descriptions in this report, chronic deciduitis of the basal plate ${ }^{27}$ and chronic chorioamnionitis are thought to be included in the analysis. Edmondson et $a l^{45}$ compared the pathological lesions in 39 cases of idiopathic preterm labor without clinical chorioamnionitis with a gestational age-matched control group and found chronic deciduitis with plasma cells in $41 \%$ of cases and in $15 \%$ of controls, respectively. The findings in the present study showing an association between chronic deciduitis with plasma cells and preterm birth cases seem to be consistent with previous observations. Collectively, all these observations suggest that a large proportion of preterm births have a non-infectious immunological component.

Another intriguing observation in this study is the relatively high frequency of villitis of unknown etiology in preterm labor $(19 \%)$ and preterm prelabor rupture of membranes $(18 \%)$ cases. This indicates that, despite the general notion that villitis of unknown etiology is largely a lesion found in term placentas, the occurrence of villitis of unknown etiology in preterm cases can vary depending on the cohorts analyzed. A study of 539 preterm and 214 term placentas also found chronic villitis in $17 \%$ of preterm deliveries without umbilical vasculitis. ${ }^{46}$ Chronic chorioamnionitis and villitis of unknown etiology seem to be different histological manifestations of the same pathological process, and thereby result in different clinical consequences depending on the anatomical regions of the placenta involved. As inflammation of the chorioamniotic membranes is present in both physiological (term) and pathological (preterm) parturition, ${ }^{8,20}$ chronic chorioamnionitis is expected to have a more profound effect than villitis of unknown etiology involving the villous part of the placenta in spontaneous preterm birth. A higher stage of chronic chorioamnionitis in preterm prelabor rupture of membranes cases than in preterm labor cases and a higher frequency of chronic chorioamnionitis in patients at term not in labor rather than term in labor represent other intriguing observations requiring further investigation.

CXCL9, CXCL10, and CXCL11 belong to a family of CXC chemokines that are involved in the migration of CXCR3 + activated T lymphocytes, and that also have anti-angiogenic activity. ${ }^{47}$ Increased expression of these chemokines has been 

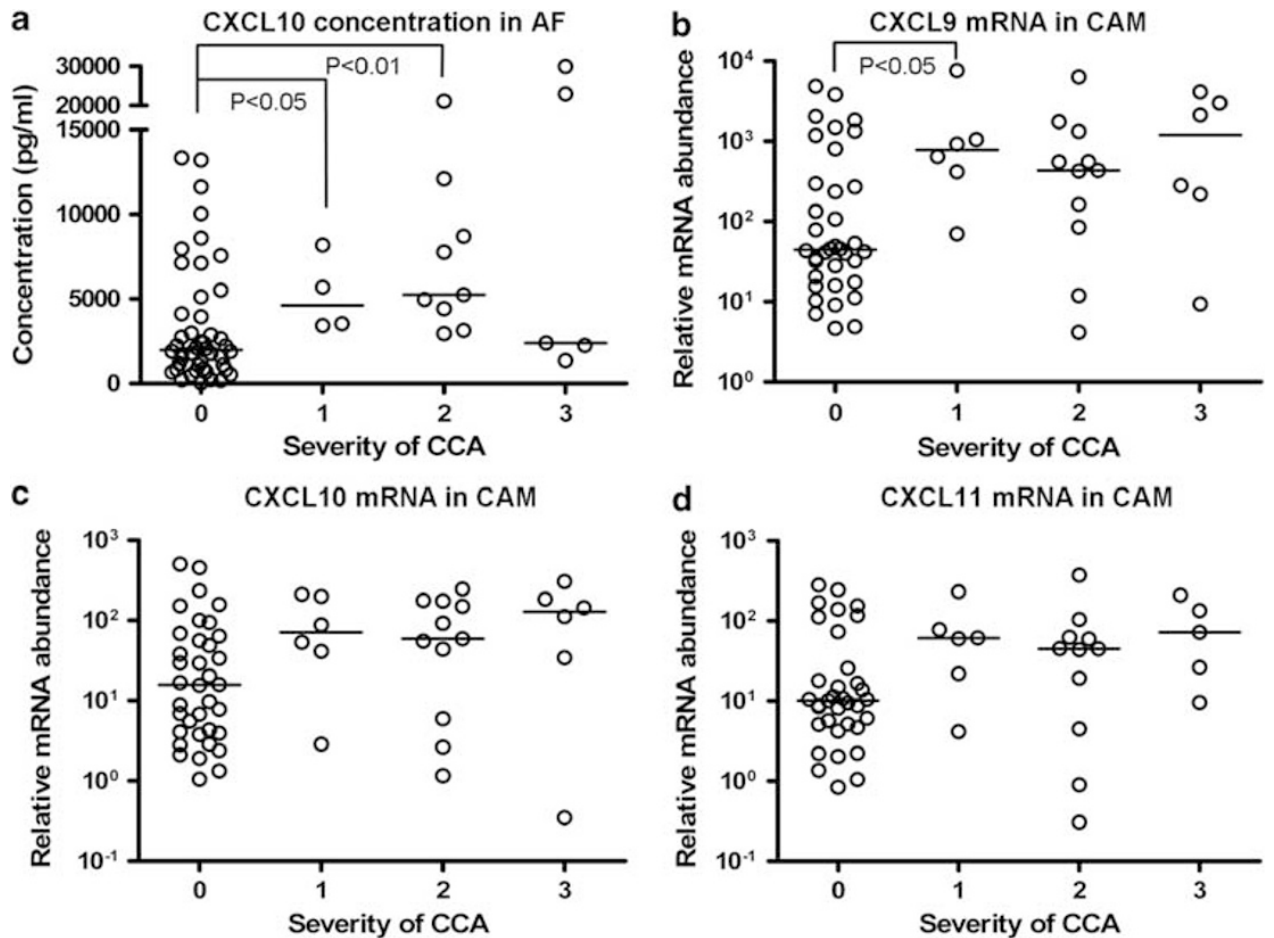

Figure 6 Comparisons of amniotic fluid (AF) CXCL10 concentration (a) and mRNA expressions of CXCL9 (b), CXCL10 (c), and CXCL11 (d) in the chorioamniotic membranes (CAM) according to the severity of chronic chorioamnionitis (CCA). Severity of chronic chorioamnionitis was graded as 1: grade 1/stage 1 inflammation, 2: grade 1/stage 2 or grade 2/stage 1 inflammation, and 3: grade 2/stage 2 inflammation. The comparisons reveal that even mild CCA is associated with significantly increased amniotic fluid CXCL10 concentration and CXCL9 mRNA expression in the chorioamniotic membranes.

described as one of the major changes taking place in either organ transplantation rejection or graftversus-host disease. ${ }^{48-51}$ Higher mRNA expressions of CXCL9, CXCL10, and CXCL11 in the chorioamniotic membranes of chronic chorioamniotic cases are quite consistent with our expectations. Pathological upregulation of CXC chemokines for CXCR3 + cells in the chorioamniotic membranes would lead to increased amniotic fluid concentration, and eventually, development of chronic chorioamnionitis by stimulating amniotropic maternal T-cell migration. A limitation of this study is that we did not measure amniotic fluid CXCL9 and CXCL11 concentrations. This was because of limited volumes of amniotic fluid available for immunoassay. However, it is noteworthy that CXCL11 concentration in the amniotic fluid was found to have predictive value for preterm birth in an analysis of 312 second-trimester transabdominal amniocentesis samples, although the placental pathology of the cases was not studied. ${ }^{52}$ We also found six cases among preterm labor and preterm prelabor rupture of membranes cases without chronic chorioamnionitis that had very high amniotic fluid CXCL10 concentrations. Re-examination of these select cases with additional CD3 immunostaining revealed acute chorioamnionitis in one case and T-cell infiltration consistent with chronic chorioamnionitis in the other four cases.
In conclusion, the findings reported in this study provide strong support for the view that chronic chorioamnionitis and increased intra-amniotic CXCL10 concentrations are an additional and important clue that may explain what has been called, thus far, 'idiopathic preterm births.' We consider that untimely birth is the result of a pathological process, and that chronic chorioamnionitis is a major immunopathological lesion of preterm birth in which even histologically mild but amniotropic T-cell inflammation is associated with a derangement of the intra-amniotic chemokine environment. Future studies of the mechanisms responsible for the perturbed T-cell chemokine regulation in the amniotic fluid and the chorioamniotic membranes are urgently needed to unveil the fundamental cause of this lesion as well as a major fraction of spontaneous preterm birth.

\section{Acknowledgements}

This work was supported in part by the Perinatology Research Branch, Division of Intramural Research, Eunice Kennedy Shriver National Institute of Child Health and Human Development, NIH, DHHS. We are grateful to the patients who agreed to participate in our studies, and to the nurses, laboratory staff, and clinicians who made this work possible. 


\section{Disclosure/conflict of interest}

The authors declare no conflict of interest.

\section{References}

1 Faye-Petersen OM. The placenta in preterm birth. J Clin Pathol 2008;61:1261-1275.

2 Romero R, Espinoza J, Kusanovic JP, et al. The preterm parturition syndrome. BJOG 2006;113:17-42.

3 Davidoff MJ, Dias T, Damus K, et al. Changes in the gestational age distribution among US singleton births: impact on rates of late preterm birth, 1992 to 2002 . Semin Perinatol 2006;30:8-15.

4 Hagberg H, Mallard C, Jacobsson B. Role of cytokines in preterm labour and brain injury. BJOG 2005;112: 16-18.

5 Romero R, Gotsch F, Pineles B, et al. Inflammation in pregnancy: its roles in reproductive physiology, obstetrical complications, and fetal injury. Nutr Rev 2007;65:S194-S202.

6 Russell RB, Green NS, Steiner CA, et al. Cost of hospitalization for preterm and low birth weight infants in the United States. Pediatrics 2007;120:e1-e9.

7 Raju TN. Epidemiology of late preterm (near-term) births. Clin Perinatol 2006;33:751-763.

8 Romero R, Espinoza J, Goncalves LF, et al. The role of inflammation and infection in preterm birth. Semin Reprod Med 2007;25:21-39.

9 DiGiulio DB, Romero R, Amogan HP, et al. Microbial prevalence, diversity and abundance in amniotic fluid during preterm labor: a molecular and culture-based investigation. PLoS ONE 2008;3:e3056.

10 Redline RW, Faye-Petersen O, Heller D, et al. Amniotic infection syndrome: nosology and reproducibility of placental reaction patterns. Pediatr Dev Pathol 2003;6:435-448.

11 Salafia CM, Weigl C, Silberman L. The prevalence and distribution of acute placental inflammation in uncomplicated term pregnancies. Obstet Gynecol 1989;73:383-389.

12 Cherouny PH, Pankuch GA, Romero R, et al. Neutrophil attractant/activating peptide-1/interleukin-8: association with histologic chorioamnionitis, preterm delivery, and bioactive amniotic fluid leukoattractants. Am J Obstet Gynecol 1993;169:1299-1303.

13 Mittal P, Romero R, Kusanovic JP, et al. CXCL6 (granulocyte chemotactic protein-2): a novel chemokine involved in the innate immune response of the amniotic cavity. Am J Reprod Immunol 2008;60: 246-257.

14 Yoon BH, Park CW, Chaiworapongsa T. Intrauterine infection and the development of cerebral palsy. BJOG 2003;110:124-127.

15 Gotsch F, Romero R, Kusanovic JP, et al. The fetal inflammatory response syndrome. Clin Obstet Gynecol 2007;50:652-683.

16 Novy MJ, Duffy L, Axthelm MK, et al. Ureaplasma parvum or Mycoplasma hominis as sole pathogens cause chorioamnionitis, preterm delivery, and fetal pneumonia in rhesus macaques. Reprod Sci 2009;16:56-70.

17 Sadowsky DW, Adams KM, Gravett MG, et al. Preterm labor is induced by intraamniotic infusions of interleukin-1beta and tumor necrosis factor-alpha but not by interleukin-6 or interleukin-8 in a nonhuman primate model. Am J Obstet Gynecol 2006;195: 1578-1589.

18 Maymon E, Romero R, Pacora P, et al. A role for the $72 \mathrm{kDa}$ gelatinase (MMP-2) and its inhibitor (TIMP-2) in human parturition, premature rupture of membranes and intraamniotic infection. J Perinat Med 2001;29:308-316.

19 Chen FC, Sarioglu N, Buscher U, et al. Lipopolysaccharide binding protein in the early diagnosis of intraamniotic infection of pregnant women with premature rupture of the membranes. J Perinat Med 2009;37:135-139.

20 Haddad R, Tromp G, Kuivaniemi H, et al. Human spontaneous labor without histologic chorioamnionitis is characterized by an acute inflammation gene expression signature. Am J Obstet Gynecol 2006; 195:394.e1-24.

21 Jacques SM, Qureshi F. Chronic chorioamnionitis: a clinicopathologic and immunohistochemical study. Hum Pathol 1998;29:1457-1461.

22 Gersell DJ, Phillips NJ, Beckerman K. Chronic chorioamnionitis: a clinicopathologic study of 17 cases. Int J Gynecol Pathol 1991;10:217-229.

23 Gersell DJ. Chronic villitis, chronic chorioamnionitis, and maternal floor infarction. Semin Diagn Pathol 1993;10:251-266.

24 Kim MJ, Romero R, Kim CJ, et al. Villitis of unknown etiology is associated with a distinct pattern of chemokine up-regulation in the feto-maternal and placental compartments: implications for conjoint maternal allograft rejection and maternal anti-fetal graft-versus-host disease. J Immunol 2009;182: 3919-3927.

25 Redline RW, Ariel I, Baergen RN, et al. Fetal vascular obstructive lesions: nosology and reproducibility of placental reaction patterns. Pediatr Dev Pathol 2004;7:443-452.

26 Redline RW, Boyd T, Campbell V, et al. Maternal vascular underperfusion: nosology and reproducibility of placental reaction patterns. Pediatr Dev Pathol 2004;7:237-249.

27 Khong TY, Bendon RW, Qureshi F, et al. Chronic deciduitis in the placental basal plate: definition and interobserver reliability. Hum Pathol 2000;31:292-295.

28 Redline RW. Villitis of unknown etiology: noninfectious chronic villitis in the placenta. Hum Pathol 2007;38:1439-1446.

29 Han YM, Romero R, Kim JS, et al. Region-specific gene expression profiling: novel evidence for biological heterogeneity of the human amnion. Biol Reprod 2008;79:954-961.

30 Raju TN, Higgins RD, Stark AR, et al. Optimizing care and outcome for late-preterm (near-term) infants: a summary of the workshop sponsored by the National Institute of Child Health and Human Development. Pediatrics 2006;118:1207-1214.

31 Wenstrom KD, Andrews WW, Bowles NE, et al. Intrauterine viral infection at the time of second trimester genetic amniocentesis. Obstet Gynecol 1998;92:420-424.

32 Liesnard C, Donner C, Brancart F, et al. Prenatal diagnosis of congenital cytomegalovirus infection: prospective study of 237 pregnancies at risk. Obstet Gynecol 2000;95:881-888.

33 Miller JL, Harman C, Weiner C, et al. Perinatal outcomes after second trimester detection of amniotic 
fluid viral genome in asymptomatic patients. J Perinat Med 2009;37:140-143.

34 Myerson D, Parkin RK, Benirschke K, et al. The pathogenesis of villitis of unknown etiology: analysis with a new conjoint immunohistochemistryin situ hybridization procedure to identify specific maternal and fetal cells. Pediatr Dev Pathol 2006;9: 257-265.

35 Kim JS, Romero R, Kim MR, et al. Involvement of Hofbauer cells and maternal $\mathrm{T}$ cells in villitis of unknown aetiology. Histopathology 2008;52: 457-464.

36 Goldenberg RL, Culhane JF, Iams JD, et al. Epidemiology and causes of preterm birth. Lancet 2008;371: 75-84.

37 Arias F, Rodriquez L, Rayne SC, et al. Maternal placental vasculopathy and infection: two distinct subgroups among patients with preterm labor and preterm ruptured membranes. Am J Obstet Gynecol 1993;168:585-591.

38 Arias F, Victoria A, Cho K, et al. Placental histology and clinical characteristics of patients with preterm premature rupture of membranes. Obstet Gynecol 1997;89:265-271.

39 McElrath TF, Hecht JL, Dammann O, et al. Pregnancy disorders that lead to delivery before the 28th week of gestation: an epidemiologic approach to classification. Am J Epidemiol 2008;168:980-989.

40 Becroft DM, Thompson JM, Mitchell EA. Placental villitis of unknown origin: epidemiologic associations. Am J Obstet Gynecol 2005;192:264-271.

41 Raju TN. The problem of late-preterm (near-term) births: a workshop summary. Pediatr Res 2006;60: 775-776.

42 Gray RF, Indurkhya A, McCormick MC. Prevalence, stability, and predictors of clinically significant behavior problems in low birth weight children at 3, 5, and 8 years of age. Pediatrics 2004;114:736-743.
43 Yoon BH, Romero R, Kim CJ, et al. Amniotic fluid interleukin-6: a sensitive test for antenatal diagnosis of acute inflammatory lesions of preterm placenta and prediction of perinatal morbidity. Am J Obstet Gynecol 1995;172:960-970.

44 Goldenberg RL, Andrews WW, Faye-Petersen O, et al. The Alabama Preterm Birth Project: placental histology in recurrent spontaneous and indicated preterm birth. Am J Obstet Gynecol 2006;195:792-796.

45 Edmondson N, Bocking A, Machin G, et al. The prevalence of chronic deciduitis in cases of preterm labor without clinical chorioamnionitis. Pediatr Dev Pathol 2009;12:16-21.

46 Salafia CM, Vogel CA, Vintzileos AM, et al. Placental pathologic findings in preterm birth. Am J Obstet Gynecol 1991;165:934-938.

47 Romagnani P, Lasagni L, Annunziato F, et al. CXC chemokines: the regulatory link between inflammation and angiogenesis. Trends Immunol 2004;25:201-209.

48 Panzer U, Reinking RR, Steinmetz OM, et al. CXCR3 and CCR5 positive T-cell recruitment in acute human renal allograft rejection. Transplantation 2004;78: 1341-1350.

49 Tan J, Zhou G. Chemokine receptors and transplantation. Cell Mol Immunol 2005;2:343-349.

50 Wysocki CA, Panoskaltsis-Mortari A, Blazar BR, et al. Leukocyte migration and graft-versus-host disease. Blood 2005;105:4191-4199.

51 Piper KP, Horlock C, Curnow SJ, et al. CXCL10-CXCR3 interactions play an important role in the pathogenesis of acute graft-versus-host disease in the skin following allogeneic stem-cell transplantation. Blood 2007;110:3827-3832.

52 Malamitsi-Puchner A, Vrachnis N, Samoli E, et al. Elevated second trimester amniotic fluid interferon gamma-inducible T-cell alpha chemoattractant concentrations as a possible predictor of preterm birth. J Soc Gynecol Investig 2006;13:25-29. 